\title{
Implant-supported crown as an alternative for missing teeth
}

\author{
${ }^{1}$ Michael Josef Kridanto K, ${ }^{1}$ Harry Laksono, ${ }^{2}$ Birgitta Dwitya Swastyayana Subiakto \\ ${ }^{1}$ Staff of Prosthodontic Dentistry Department \\ ${ }^{2}$ Post Graduate Student of Prosthodontic Dentistry Department \\ Faculty of Dental Medicine, Universitas Airlangga \\ Surabaya, Indonesia \\ Corresponding author: Michael Josef Kridanto K, email: josef_310563@yahoo.com
}

\begin{abstract}
The use of dental implants to support fixed or removable restoration is widely used as treatment modality. The advantages are increased retention, chewing ability, and easy access to oral hygiene procedures. A missing tooth that needs to be replaced completely can be restored using an implant-supported crown. The aim of this study is to rehabilitate maxillary partial edentulous with implant supported crown. A 66-year-old male patient came to the clinic, wanted to replace partial edentulous after extraction of 13 and 14, needed fixed restoration in order to eat and chew well, and expected high aesthetic result. This patient had experienced using implants to replace teeth loss in other regions. Patient wanted to have implant treatment with fixed restoration because he had experienced with the same treatment before in another region, so the prosthodontic treatment option was using implant supported crowns. Implant supported crown can be an option to replace partial edentulous.
\end{abstract}

Keyword: implant supported crown, fixed restoration, dental implant

\section{INTRODUCTION}

Dental implants, also known as oral or endosseous implants, have been used to replace missing teeth for more than half a century. Dental implant restoration has been considered to be one of the most reliable methods for treating partial or full edentulism. They are considered to be an important contribution to dentistry as they have revolutionized the way by which missing teeth are replaced with a high success rate. This success depends on the ability of the implant material to integrate with the surrounding tissue. However, this integration is influenced by several factors, such as implant materialS, bone quality and quantity, and the implant loading condition. ${ }^{1}$

Bone graft is frequently accompanied with dental implant surgery. Various types of bone graftmaterials are used such as the autogenous bone, allogenic bone, xenogenic bone, and synthetic materials. The most frequently used surgical methods for bone grafts are guided bone regeneration (GBR), block bone graft (BBG), sinus lifting via lateral window, and bone-added osteotome technique. GBR procedure needs bone graft materials and a membrane for selective occlusiveness. Bone graft materials can be used solely or mixed together in different proportions. ${ }^{2}$ Similar cases of bone defects can be treated differently according to the surgeon's preference.

The aim of this study is to rehabilitate maxillary partial edentulous with implant supported crown.

\section{CASE}

A66-year-old male patient came to the clinic wanted to replace partial edentulous after extrac- tion of 13 and 14. This patient wanted fixed restoration in order to eat and chew well and expected high aesthetic result.

This patient had experienced using implants to replace teeth loss in other regions. In this case,

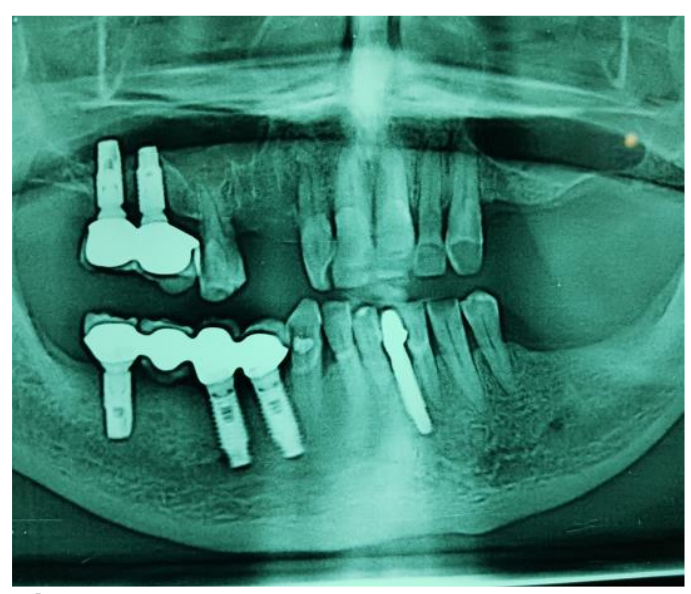

Fig $1 \mathrm{X}$-ray panoramic

patient lost his canine and first premolar on the right maxilla. Patient wanted to have implant treatment with fixed restoration because he had experienced with the same treatment before in another regions, so the prosthodontic treatment option was using implant supported crowns.

\section{MANAGEMENT}

The first stage when the patient came for a consultation was taking X-ray. The X-ray shows a defect due to tooth extraction 13 and 14, which was done by adding bone graft in the area. On the next visit an implant placement $\varnothing 3.3 \times 8 \mathrm{~mm}$ (Straumann, Switzerland) was followed by bone grafting and membrane (Straumann, Switzerland) 
in areas 13 and 14 . Then a healing screw was placed to help guide the gingiva in the proper way to heal. Then, wound closure was performed by tension-free repositioning and suturing of the flap.

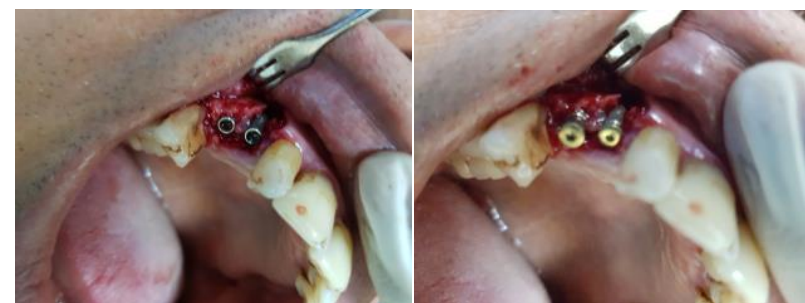

Fig 2A Two bone level implant fixture were inserted at region 13 and 14; $\mathbf{B}$ bone graft application, wound closure by tension-free repositioning and suturing of the flap

After 6 months, the healing screw was opened and a screw abutment was placed, which is the part that screws into the implant and will support the crown. Once the abutment was placed, we took another impression of the abutment for each replacement tooth. Then the patient got a temporary crown while the tissues continued to heal and form around the artificial tooth as with the natural teeth. The patient wore the temporary crown for four to six weeks. During this time, the permanent crown would be made. Then, the final stage of the procedure was placing the crown. Crowns were screwed into the abutment to this patient.

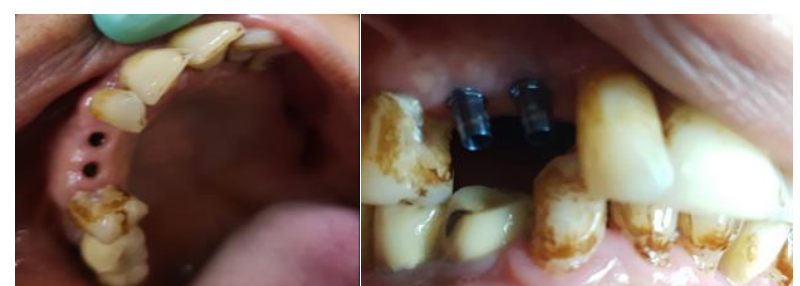

Fig 3A Healing screws were opened 6 months after implants insertion; B two cemented abutments were engaged to the implants

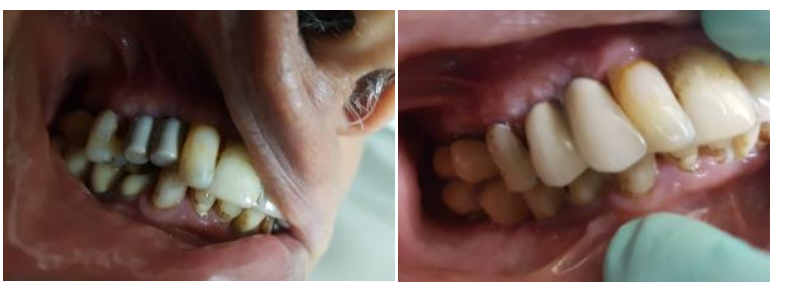

Fig 4A Two plastic protective caps were used to protect the abutments during laboratory process; $\mathbf{B} 2$ porcelainfused to metal were chosen as final restorations.

\section{DISCUSSION}

Dental implants have been used to replace missing teeth for more than half a century. Dental implant considered to be an important contribution to dentistry by which missing teeth are replaced with a high success rate. This success depends on the ability of the implant material to integrate with the surrounding tissue.

Placement of implants requires sufficientbone volume and biologic quality. Some cases need socket preservation or ridge preservation. In this case, there is resorption of the edentulous ridge post extraction which make socket preservation or ridge preservation necessary.

These procedures involve filling the socket with bone or bone substitute material, with or without membrane. The aim of ridge preservations are filling the socket (wound care), preservation of ridge volume (ridge preservation), and new bone formation (osteogenesis). Dental implant is considered as the most reliable and convenient treatment for partial and full edentulism. Long-term follow-up of the implants showed successful survival rate of over $90 \%$. $^{3}$

In several studies, $50.3 \%$ of the patients required bone graft during implant surgery. The anterior maxillary area required more than $77 \%$ bone graft. Because of the high esthetic demands in the anterior maxilla, bone augmentation was performed even though there was no bone fenestration or dehiscence. ${ }^{3}$ Autogenous bone graft in exposed threads of the implant was suggested as a golden standard. After autogenous bone graft, xenogenic bone and absorbable membrane were used for additional augmentation for long-term esthetic results. At least 1.5 $2 \mathrm{~mm}$ of buccal bone is required for esthetic results in the anterior maxilla. ${ }^{4}$

In this case, bone grafting was decided because of the presence of thin labial plate in areas 13 and 14 . The indications for GBR are dehiscence or fenestration wound or thin labial plate which was expected to resorb during healing. If the width of the residual alveolarbone in the anterior maxilla was less than $3 \mathrm{~mm}$, BBG was performed. BBG was performed in the anterior maxilla most frequently than in any other sites. ${ }^{5}$

During GBR procedures, xenogenic bone with/ without autogenous bone was the most commonly used. The advantages of the xenogenic bone include slow bone resorption during the healing phase and its wide availability. Although there was no bone dehiscence, xenogenic bone was recommended to graft for the augmentation of the labial bone. In this study, absorbable membrane (Straumann, Switzerland) was used for GBR procedure. ${ }^{5}$

In this case, the retention of the restoration relies on the retaining screw. Nevertheless, the restoration can be removed and/or replaced when required, without damage or need a new restora- 
tion. The adaptation between the restoration and the underlying implant is usually better than that in the case of its cement-retained counterpart. It can be used when the vertical restorative space is limited as the retention depends on the screw, but is contra-indicated when mouth opening is limited, as the use of the different tools required for screwing and torqueing the screws may not be possible. ${ }^{2}$

However, the use of a screw-retained restoration may be considered when the implant platform is situated deep sub-mucosally, as complete removal of cement is not always possible when a cement-retained restoration is used. The screw type is not indicated when the screw hole is point- ed at the labial surface as this compromises the aesthetics. Hence, the implant should be placed in its optimal position and angulation to avoid negative effects on aesthetics, otherwise an angled abutment may provide an acceptable alternative.

So, it was concluded that Implant can replace missing teeth to restore masticatory function and aesthetic for the patient. Bone graft was necessary to augment the defect areas during implant surgery. The success of any implant supported restoration is dependent on the interaction between the patient and the dental personnel. Maintaining good oral hygiene and committing to regular check-ups are the responsibility of the patient.

\section{REFERENCES}

1. Zupnik J, Kim S, Ravens D, Karimbux N, Guze K. Analysis. J Periodontol 2011;82:1390-5.

2. Warreth A. Dental implants : an overview. Dent Updat 2018; 44: 596-620.

3. Ribeiro CG, Bittencourt TC, Ferreira CF, Maria N, Picorelli S. An alternative approach for augmenting the anterior maxilla using autogenous free gingival bone graft for implant retained prosthesis. J Oral Implantol 2014;XL(2):183-7.

4. Chen ST, Buser FD, Dent PM. Esthetic outcomes following immediate and early implant placement in the anterior maxilla — a systematic review. Int J Oral Maxillofac Implant 2014;29(1):187-95.

5. Cha H, Kim J, Hwang J, Ahn K. Frequency of bone graft in implant surgery. Maxillofac Plast Reconstr Surg [Internet]. 2016;38(19):2-5. Available from: http://dx.doi.org/10.1186/s40902-016-0064-2. 